\title{
Synthesis of 6-(arylthio)phenanthridines by cyclization reaction of 2-isocyanobiphenyls with thiols
}

\author{
Changpeng Wu, Yuhan Zhou,* Xiaoliang Dong, and Jingping Qu \\ State Key Laboratory of Fine Chemicals, School of Pharmaceutical Science and Technology, \\ Dalian University of Technology, Dalian 116024, P.R. China \\ E-mail:zhouyh@dl.cn
}

DOI: $\underline{\text { http://dx.doi.org/10.3998/ark.5550190.p009.428 }}$

\begin{abstract}
An efficient method for the synthesis of 6-(arylthio)phenanthridines by tert-butyl peroxybenzoate (TBPB)-promoted cyclization reaction of 2-isocyanobiphenyls with thiols is developed. A radical pathway is proposed and evidenced for the reaction mechanism. It tolerates a wide range of substrates and represents a practical approach to 6-arylthiophenanthridines.
\end{abstract}

Keywords: Phenanthridines, thiols, isocyanides, cyclization, radical reactions

\section{Introduction}

Phenanthridines are common constituents of some alkaloids and potential pharmaceuticals (Figure 1), and these heterocycles show biological activities, ${ }^{1-5}$ such as antitumor, antileukemic, antiviral, and antifungal properties. ${ }^{6-11}$ In addition, they have excellent optical and electronic properties in the fields of functional materials. ${ }^{12,13}$

In recent years, the synthesis of phenanthridine derivatives via the radical addition and cyclization of 2-isocyanobiphenyls has received much attention. Several radical precursors have been used, such as boronic acids, ${ }^{14} \mathrm{CF}_{3}$ reagents, ${ }^{15-17}$ aldehydes, ${ }^{18}$ acyl peroxides, ${ }^{19}$ simple alkanes, ${ }^{20}$ halides, ${ }^{21,22}$ diphenylphosphine oxide, ${ }^{23}$ arenesulfonyl chlorides, ${ }^{24} \alpha$-oxocarboxylic acids and hydrazines, ${ }^{25,26}$ and as a result the corresponding 6-functionalized phenanthridine derivatives were prepared. However, only a few examples of the construction of 6-arylthiosubstituted phenanthridines have been reported. The formation of 6-arylthiophenanthridines from 2-isocyanobiaryls and disulfides was first described by Han and Pan. ${ }^{27}$ However, this method requires a large excess (6 equiv) of peroxide and non-atom economic disulfides. And 6arylthiophenanthridines can be obtained by the reaction of 2-biaryl isothiocyanates and diaryliodonium salts, which are costly. ${ }^{28}$ In addition, they can also be obtained from the nucleophilic substitution of 6-chlorophenanthridines with thiophenols. ${ }^{29,30}$ Methods for the 
construction of 6-arylthiophenanthridines are still desired. To this end, we report the radical addition and cyclization of 2-isocyanobiphenyls with thiophenols to prepare 6arylthiophenanthridines.<smiles>CCSCCc1cc2ccc3c4cc(C)c(C)cc4c[n+](C)c3c2cc1C</smiles><smiles>Cc1cc2c[n+](C)c3c(C)c(C)ccc3c2cc1C</smiles><smiles>C=C(C)c1cccc(I)c1</smiles><smiles></smiles>

1<smiles>CC[n+]1c(-c2ccccc2)c2cc(I)ccc2c2ccc(C)cc21</smiles>

Figure 1. Biologically active phenanthridines.

\section{Results and Discussion}

Initially, 2-isocyano-4'-methoxybiphenyl (1a) and p-toluenethiol (2a) were selected as model substrates for optimization of the reaction conditions (Table 1). When a mixture of 1a and 2a with $t$-BuOK was heated at $120{ }^{\circ} \mathrm{C}$ in xylene for $5 \mathrm{~h}$ under argon, no product was obtained (Table 1, entry 1). The addition of azobisisobutyronitrile (AIBN) provided the product 3a, although in only $19 \%$ yield (entry 2). Several other radical initiators were then examined. Phthaloyl peroxide, 2,3-dichloro-5,6-dicyano-1,4-benzoquinone (DDQ) and $\mathrm{Na}_{2} \mathrm{~S}_{2} \mathrm{O}_{8}$ did not give the required product (Table 1, entries 6, 8 and 9). Interestingly, benzoyl peroxide (BPO), $t$ butyl peroxide (DTBP) and dicumyl peroxide (DCP) all promoted the reaction, in 29\%, 30\% and $33 \%$ yields respectively, while tert-butyl peroxybenzoate (TBPB) afforded a 53\% yield (entries 3-7). Therefore, TBPB was chosen for further study. We found that an increase in the amount of TBPB slightly decreased the yield (Table 1, entry 10).

A base is also essential for this reaction. Without a base, 3a was obtained in only $34 \%$ yield (Table 2, entry 1). Improved yields were observed when inorganic bases such as $\mathrm{K}_{2} \mathrm{CO}_{3}, \mathrm{Cs}_{2} \mathrm{CO}_{3}$ and $\mathrm{NaOH}$ were used (Table 2, entries 2-4). When organic bases were examined, DBU and $\mathrm{NEt}_{3}$ were found to be unfavorable (Table 2, entries 5 and 6), while $t$-BuOK and MeONa gave 
moderate yields (Table 2, entries 8 and 9). Further increase of the amount of base resulted in a decrease in the yield (Table 2, entry 10 ).

Table 1. Optimization of oxidant for the reaction ${ }^{a}$<smiles>CCc1ccc(-c2ccccc2C)cc1</smiles>

\begin{tabular}{cccccc}
\hline Entry & Initiator & ${\text { Yield }(\%)^{b}}^{b}$ & Entry & Initiator & Yield $(\%)^{b}$ \\
\hline 1 & none & ND & 6 & Phthaloyl peroxide & ND $^{c}$ \\
2 & AIBN & 19 & 7 & TBPB & 53 \\
3 & BPO & 29 & 8 & DDQ & ND \\
4 & DTBP & 30 & 9 & $\mathrm{Na}_{2} \mathrm{~S}_{2} \mathrm{O}_{8}$ & ND \\
5 & DCP & 33 & $10^{d}$ & TBPB & 48 \\
\hline
\end{tabular}

${ }^{a}$ Reaction conditions: 1a $(0.3 \mathrm{mmol}), \mathbf{2 a}(0.36 \mathrm{mmol})$, initiator $(0.9 \mathrm{mmol}), t$-BuOK $(0.3 \mathrm{mmol})$, xylene $(3 \mathrm{~mL}), 120{ }^{\circ} \mathrm{C}$ for $5 \mathrm{~h}$ under nitrogen. ${ }^{b}$ Yields were determined by ${ }^{1} \mathrm{H}$ NMR with 1,2dichloroethane as an internal standard. ${ }^{c} \mathrm{ND}$ : not detected. ${ }^{d}$ TBPB $1.8 \mathrm{mmol}$.

Furthermore, the effect of solvents was explored. Solvents also play an important role in the reaction. When the reaction was carried out in polar solvents, such as DMF, DMSO and NMP, the yield of the desired product decreased dramatically (Table 2, entries 11-13). Toluene and chlorobenzene gave similar yields compared with xylene (Table 2, entries 15 and 16).

Table 2. Optimization of base and solvent for the reaction ${ }^{a}$

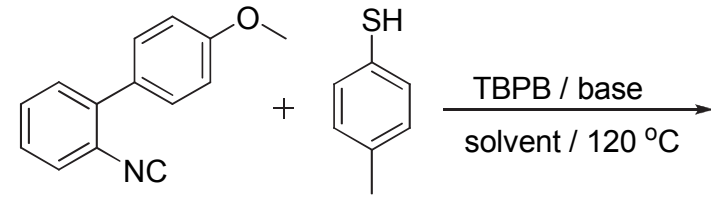

1a<smiles>COc1ccc2c(c1)c(Sc1ccc(C)cc1)nc1ccccc12</smiles>

$3 a$

\begin{tabular}{cccccccc}
\hline Entry & Base & Solvent & $\begin{array}{c}\text { Yield }^{b} \\
(\%)\end{array}$ & Entry & Base & Solvent & $\begin{array}{c}\text { Yield }^{b} \\
(\%)\end{array}$ \\
\hline 1 & none & xylene & 34 & 9 & $\mathrm{MeONa}$ & xylene & 54 \\
2 & $\mathrm{~K}_{2} \mathrm{CO}_{3}$ & xylene & 43 & 10 & $\mathrm{MeONa}$ & xylene & 28 \\
3 & $\mathrm{NaOH}$ & xylene & 44 & 11 & $\mathrm{MeONa}$ & $\mathrm{DMF}$ & 25 \\
4 & $\mathrm{Cs}_{2} \mathrm{CO}_{3}$ & xylene & 35 & 12 & $\mathrm{MeONa}$ & $\mathrm{DMSO}$ & $\mathrm{ND}$ \\
5 & $\mathrm{DBU}^{c}$ & xylene & 10 & 13 & $\mathrm{MeONa}$ & $\mathrm{NMP}$ & 34 \\
6 & $\mathrm{NEt}_{3}$ & xylene & 33 & 14 & $\mathrm{MeONa}$ & trifluorotoluene & 39 \\
\hline
\end{tabular}


Table 2 (continued)

\begin{tabular}{cccccccc}
\hline Entry & Base & Solvent & $\begin{array}{c}\text { Yield }^{b} \\
(\%)\end{array}$ & Entry & Base & Solvent & $\begin{array}{c}\text { Yield }^{b} \\
(\%)\end{array}$ \\
\hline 7 & $\mathrm{NaOAc}$ & xylene & 46 & 15 & $\mathrm{MeONa}$ & chlorobenzene & 53 \\
8 & $t$-BuOK & xylene & 53 & 16 & $\mathrm{MeONa}$ & toluene $^{d}$ & 52 \\
\hline
\end{tabular}

${ }^{a}$ Reaction conditions: 1a $(0.3 \mathrm{mmol}), \mathbf{2 a}(0.36 \mathrm{mmol})$, TBPB $(0.9 \mathrm{mmol})$, base $(0.3 \mathrm{mmol})$, solvent $(3 \mathrm{~mL}), 120{ }^{\circ} \mathrm{C}$ for $5 \mathrm{~h}$ under nitrogen. ${ }^{b}$ Yields were determined by ${ }^{1} \mathrm{H}$ NMR with 1,2dichloroethane as an internal standard. ${ }^{c} \mathrm{MeONa}(0.6 \mathrm{mmol}) .{ }^{d}$ The reaction was carried out in a sealed tube.

After the establishment of the optimal reaction conditions, various thiophenols and also ethylthiol were tested for this oxidative thiolation / cyclization reaction with 2-isocyano-4'methoxybiphenyl. As is evident from Table 3, ortho and para substituents on thiophenol ring did not have any significant influence on the yield of the reaction $(45 \%-68 \%$ for 3a-3d, Table 3 , entries 1-4). However, fluorine substitution decreased the yield to 30\% (Table 3, entry 5). Not only could thiophenols give moderate yields, but also ethylthiol gave a yield of $45 \%$ (Table 3 , entry 6).

Table 3. Reaction of 2-isocyano-4'-methoxybiphenyl with various thiols ${ }^{a}$

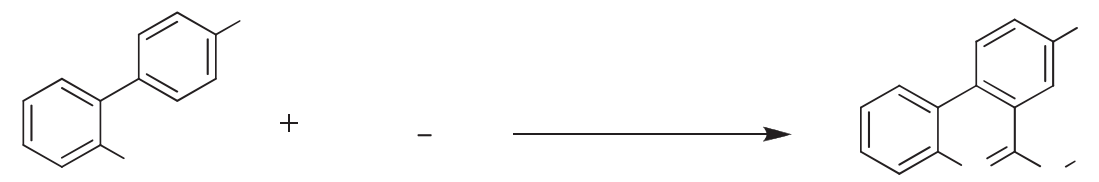

\begin{tabular}{cccc}
\hline Entry & $\mathrm{R}^{1}$ & Product & Yield/\% ${ }^{b}$ \\
\hline 1 & $\mathbf{3 a}$ & 53 \\
2 & $\mathbf{3 b}$ & 58 \\
3 & $\mathbf{3 c}$ & 45 \\
4 & $\mathbf{3 d}$ & 68 \\
5 & $\mathrm{3e}$ & 30 \\
\hline
\end{tabular}

${ }^{a}$ Reaction conditions: 2-isocyano-4'-methoxy-biphenyl (0.3 mmol), $\mathrm{R}^{1}-\mathrm{SH}$ (0.36 mmol), TBPB $(0.9 \mathrm{mmol}), \mathrm{MeONa}(0.3 \mathrm{mmol})$, solvent $(3 \mathrm{~mL}), 120{ }^{\circ} \mathrm{C}$ for $5 \mathrm{~h}$ under nitrogen. ${ }^{b}$ Isolated yield.

A broad range of 2-isocyanobiphenyls were then investigated under standard reaction conditions; the results are summarized in Table 4. The electronic effect of the substituents on the 
aromatic ring with the isocyano group was unnoticeable. The functional groups, such as acetyl, ester, cyano, $t$-butyl, fluorine and chlorine were all tolerated well, and the desired products were obtained in moderate yields (Table 4, entries 1-7). Next, a variety of substituted thiophenols were subjected to the reaction. Once again, 4-bromothiophenol, thiophenol, 3-methoxybenzenethiol, 4-toluenethiol, 2-naphthalenethiol and 4-fluorothiophenol worked well, providing the desired products in moderate yields. In addition, reactions with different isocyanides also proceeded smoothly, which furnished the desired product 3n, 3o, 3q, 3r, 3s and 3t in 41\%, 55\%, $29 \%, 40 \%, 41 \%$ and $60 \%$ yields, respectively. To investigate the regioselectivity of the cyclization, 2-isocyanobiphenyl bearing a $m$-methoxy (Table 4 , entry 15) was investigated, which resulted in a mixture of two regioisomers in a ratio of 3:2. Gratifyingly, 3-(2-isocyanophenyl)pyridine gave the corresponding products in $79 \%$ yield and 3:2 regioselectivity (Table 4 , Entry 16).

Table 4. Preparation of phenanthridine derivatives ${ }^{a}$

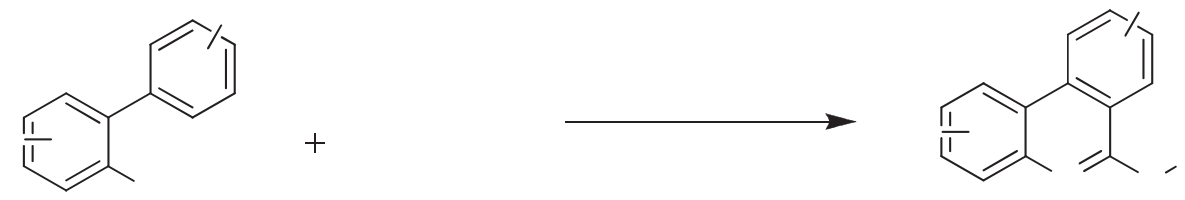

Entry Isocyanides (1)

Footnotes: see foot of Table continuation 
Table 4 (continued)

\begin{tabular}{|c|c|c|c|c|}
\hline Entry & Isocyanides (1) & $\mathrm{R}^{1} \mathrm{SH}(\mathbf{2})$ & Product (3) & Yield (\%) ${ }^{b}$ \\
\hline 7 & & $\mathrm{PhSH}$ & & 60 \\
\hline 8 & $1 \mathrm{~h}$ & $4-\mathrm{MeC}_{6} \mathrm{H}_{4} \mathrm{SH}$ & & 41 \\
\hline 9 & 1e & $4-\mathrm{MeC}_{6} \mathrm{H}_{4} \mathrm{SH}$ & & 55 \\
\hline 10 & $1 b$ & $4-\mathrm{MeC}_{6} \mathrm{H}_{4} \mathrm{SH}$ & & 55 \\
\hline 11 & & $4-\mathrm{BrC}_{6} \mathrm{H}_{4} \mathrm{SH}$ & & 29 \\
\hline 12 & & $4-\mathrm{BrC}_{6} \mathrm{H}_{4} \mathrm{SH}$ & & 40 \\
\hline 13 & & & & 41 \\
\hline 14 & 11 & $4-\mathrm{BrC}_{6} \mathrm{H}_{4} \mathrm{SH}$ & & 60 \\
\hline 15 & $1 \mathrm{~m}$ & $\mathrm{PhSH}$ & $\mathbf{3} \mathbf{u}+\mathbf{3} \mathbf{u}^{\prime}(3$ & 50 \\
\hline 16 & 1n & $\mathrm{PhSH}$ & $\mathbf{3 v}+\mathbf{3} \mathbf{v}^{\prime}(3:$ & 79 \\
\hline
\end{tabular}

${ }^{a}$ Reaction conditions: Isocyanides (1) $(0.3 \mathrm{mmol})$, Thiophenols $(2)(0.36 \mathrm{mmol})$, TBPB (0.9 mmol), MeONa $(0.3 \mathrm{mmol})$, solvent $(3 \mathrm{~mL}), 120{ }^{\circ} \mathrm{C}$ for $5 \mathrm{~h}$ under nitrogen. ${ }^{b}$ Isolated yield.

${ }^{c}$ The ratio of isolated products 
To probe the mechanism, the control experiment with the radical scavenger was carried out (Scheme 1). When the radical scavenger (2,2,6,6-tetramethylpiperidin-1-yl)oxyl (TEMPO) was added to the reaction mixture, none of the desired product was detected (Scheme 1 a). This result provides evidence for a free radical mechanism. The reaction did not proceed in the absence of TBPB (Table 1, entry 1), which indicated that TBPB plays the role of reaction promoter. In addition, we performed the reaction of 2-isocyano-4'-methoxybiphenyl (1a) with 1,2diphenyldisulfane under the standard conditions, only giving a 30\% yield (Scheme 1 b). So we excluded the possibility that thiophenol was oxidized to 1,2-diphenyldisulfane on the major pathway in the reaction. Accordingly, a possible mechanism is illustrated in Scheme 2. Firstly, the homolytic cleavage of TBPB produces a $t$-butoxy radical (A) and a benzoate radical (B) which abstracts the $S$-H-atom from the thiophenol to give thiophenyl radical (C). Then, addition of the radical species (C) to the isonitrile produces another radical intermediate (D). Subsequently, intermediate (D) cyclizes to generate the cyclohexadienyl radical (E). Further reaction of the radical $\mathbf{E}$ has two plausible directions. One is further oxidation by the benzoate radical or $t$-butoxyl radical to give the intermediate $(\mathbf{F})$, and then the phenanthridine $(\mathbf{G})$ is delivered after deprotonation. The other is that the benzoate radical or the $t$-butoxyl radical will abstract the $\mathrm{H}$-atom from the intermediate $(\mathbf{E})$, resulting in the product $(\mathbf{G})$.<smiles>CCc1ccc(-c2ccccc2C)cc1</smiles><smiles>Cc1ccccc1</smiles>

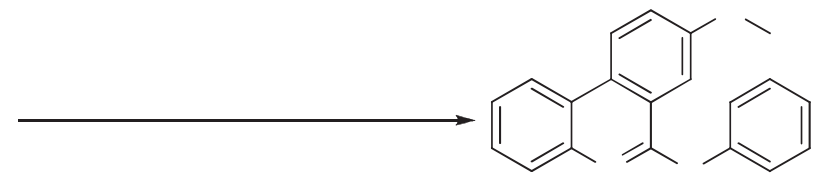<smiles>COc1ccccc1-c1ccccc1</smiles>

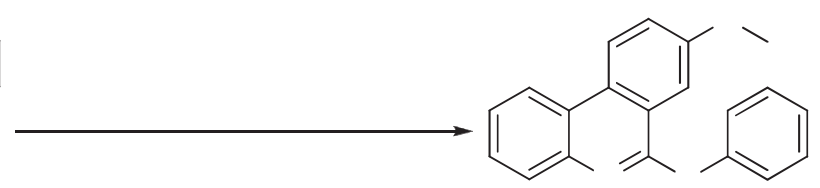

Scheme 1. The control experiments for the mechanism. 


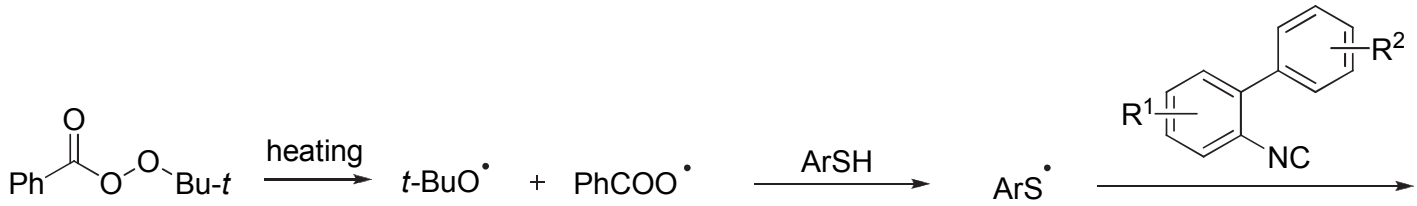

A

B

C

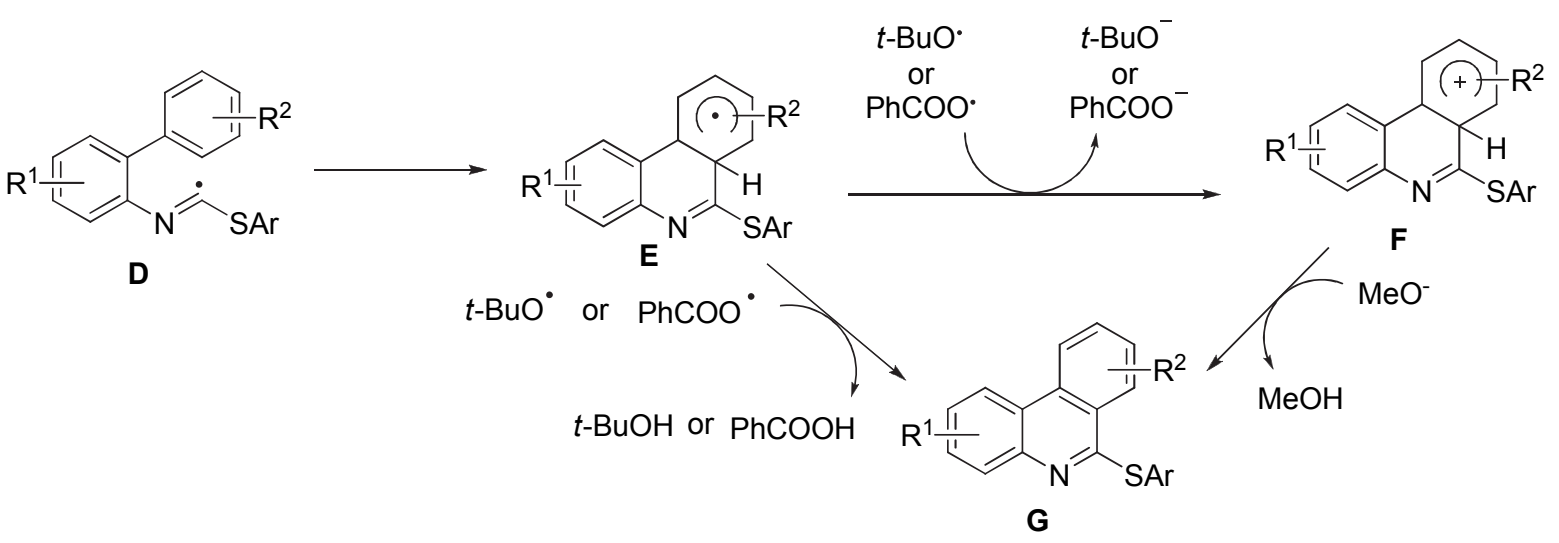

Scheme 2. Proposed mechanism for the phenanthridine formation.

In conclusion, we have developed a TBPB-promoted phenanthridinylation of simple thiol sources with 2-isocyanobiphenyls. A radical pathway was proposed and evidenced for the reaction mechanism. The functional group, such as acetyl, ester, cyano, $t$-butyl, fluorine and chlorine were all tolerated well, and the desired products were obtained in moderate yields. This represents a practical approach to access 6-arylthiophenanthridines.

\section{Experimental Section}

General. Melting points were measured on a Novel X-5 melting point instrument. All ${ }^{1} \mathrm{H}$ NMR $(400 \mathrm{MHz})$ and ${ }^{13} \mathrm{C}$ NMR $(125 \mathrm{~Hz})$ spectra were measured in $\mathrm{CDCl}_{3}$ and recorded on Bruker Avance II $400\left({ }^{1} \mathrm{H}\right.$ NMR) or Bruker Avance III $500\left({ }^{13} \mathrm{C}\right.$ NMR) spectrometer with chemical shifts reported as ppm (with TMS as an internal standard). For chromatography, neutral aluminum oxide or silica gel was employed. HRMS were conducted on GCT mass spectrometer (EI) or a LTQ Orbitrap XLTM spectrometer in positive electrospray ionization (ESI+) mode. 2isocyanobiphenyls (1) were synthesized via a three step route according to the paper previously. ${ }^{17}$ The newly synthesized 6-arylthiophenanthridines compounds are described below.

2-Isocyano-4'-methoxybiphenyl (1a). ${ }^{16}$ Pale white solid; yield $78 \%, 1438 \mathrm{mg}$; mp: $66-67{ }^{\circ} \mathrm{C}$ (lit. $\left.{ }^{16} \mathrm{mp}: 56-57{ }^{\circ} \mathrm{C}\right) .{ }^{1} \mathrm{H}$ NMR $\left(400 \mathrm{MHz}, \mathrm{CDCl}_{3}\right) \delta_{H} 7.46-7.40(5 \mathrm{H}, \mathrm{m}, \mathrm{ArH}), 7.34-7.32$ $(1 \mathrm{H}, \mathrm{m}, \mathrm{ArH}), 7.01\left(2 \mathrm{H}, \mathrm{d},{ }^{3} \mathrm{~J}_{\mathrm{HH}} 8.8 \mathrm{~Hz}, \mathrm{ArH}\right), 3.86\left(3 \mathrm{H}, \mathrm{s}, \mathrm{OCH}_{3}\right)$.

2-Isocyanobiphenyl (1b). ${ }^{\mathbf{1 6}}$ Green liquid; yield 83\%, $1188 \mathrm{mg} ;{ }^{1} \mathrm{H}$ NMR (400 MHz, $\left.\mathrm{CDCl}_{3}\right) \delta_{H}$ $7.52-7.40(8 \mathrm{H}, \mathrm{m}, \mathrm{ArH}), 7.36\left(1 \mathrm{H}, \mathrm{d},{ }^{3} \mathrm{~J}_{\mathrm{HH}} 7.8 \mathrm{~Hz}, \mathrm{ArH}\right)$. 
1-(2'-Isocyano-[1,1'-biphenyl]-4-yl)ethanone (1c). ${ }^{20}$ White solid; yield 82\%, $1449 \mathrm{mg}$; mp: 93 - 94 ${ }^{\circ} \mathrm{C} .{ }^{1} \mathrm{H}$ NMR $\left(400 \mathrm{MHz}, \mathrm{CDCl}_{3}\right) \delta_{H} 8.07\left(2 \mathrm{H}, \mathrm{d},{ }^{3} J_{\mathrm{HH}} 8.3 \mathrm{~Hz}, \mathrm{ArH}\right), 7.62\left(2 \mathrm{H}, \mathrm{d},{ }^{3} J_{\mathrm{HH}} 8.4\right.$ $\mathrm{Hz}, \mathrm{ArH}), 7.50-7.43$ (4H, m, ArH), $2.65\left(3 \mathrm{H}, \mathrm{s}, \mathrm{COCH}_{3}\right)$.

Methyl 2'-isocyano-[1,1'-biphenyl]-4-carboxylate (1d). ${ }^{16}$ White solid; yield 84\%, $1592 \mathrm{mg}$; mp: $146-147{ }^{\circ} \mathrm{C}\left(\right.$ lit. $\left.{ }^{16} \mathrm{mp}: 136-138{ }^{\circ} \mathrm{C}\right) .{ }^{1} \mathrm{H}$ NMR $\left(400 \mathrm{MHz}, \mathrm{CDCl}_{3}\right) \delta_{H} 8.15\left(2 \mathrm{H}, \mathrm{d},{ }^{3} J_{\mathrm{HH}}\right.$ 8.1Hz, ArH), $7.59\left(2 \mathrm{H}, \mathrm{d},{ }^{3} J_{\mathrm{HH}} 8.1 \mathrm{~Hz}, \mathrm{ArH}\right), 7.52-7.40(4 \mathrm{H}, \mathrm{m}, \mathrm{ArH}), 3.95\left(3 \mathrm{H}, \mathrm{s}, \mathrm{COOCH}_{3}\right)$. 4'-Chloro-2-isocyanobiphenyl (1e). ${ }^{16}$ Pale yellow solid; yield $72 \%, 1226 \mathrm{mg}$; mp: $85-89{ }^{\circ} \mathrm{C}$ (lit. $\left.{ }^{16} \mathrm{mp}: 85-89{ }^{\circ} \mathrm{C}\right) .{ }^{1} \mathrm{H}$ NMR $\left(400 \mathrm{MHz}, \mathrm{CDCl}_{3}\right) \delta_{H} 7.50-7.39(8 \mathrm{H}, \mathrm{m}, \mathrm{ArH})$.

4'-Fluoro-2-isocyanobiphenyl (1f). ${ }^{\mathbf{1 8}}$ Green liquid; yield 83\%, $1308 \mathrm{mg} ;{ }^{1} \mathrm{H}$ NMR (400 MHz, $\left.\mathrm{CDCl}_{3}\right) \delta_{H} 7.50-7.44(4 \mathrm{H}, \mathrm{m}, \mathrm{ArH}), 7.41-7.37(2 \mathrm{H}, \mathrm{m}, \mathrm{ArH}), 7.17\left(2 \mathrm{H}, \mathrm{d},{ }^{3} J_{\mathrm{HH}} 8.7 \mathrm{~Hz}, \mathrm{ArH}\right)$.

2'-Isocyano-[1,1'-biphenyl]-4-carbonitrile (1g). ${ }^{16}$ White solid; yield 62\%, $1001 \mathrm{mg}$; mp: 121 $122{ }^{\circ} \mathrm{C} .{ }^{1} \mathrm{H}$ NMR $\left(400 \mathrm{MHz}, \mathrm{CDCl}_{3}\right) \delta_{H} 7.78\left(2 \mathrm{H}, \mathrm{d},{ }^{3} J_{\mathrm{HH}} 8.4 \mathrm{~Hz}, \mathrm{ArH}\right), 7.64\left(2 \mathrm{H}, \mathrm{d},{ }^{3} J_{\mathrm{HH}} 8.5\right.$ $\mathrm{Hz}, \mathrm{ArH}), 7.56-7.38$ (4H, m, ArH).

4'-(t-Butyl)-2-isocyano-biphenyl (1h). ${ }^{19}$ Green oil; yield 84\%, $1579 \mathrm{mg} ;{ }^{1} \mathrm{H}$ NMR $(400 \mathrm{MHz}$, $\left.\mathrm{CDCl}_{3}\right) \delta_{H} 7.50-7.34(8 \mathrm{H}, \mathrm{m}, \mathrm{ArH}), 1.37(9 \mathrm{H}, \mathrm{s}, t-\mathrm{Bu})$.

1-(2-Isocyanophenyl)naphthalene (1i). Green solid; yield $78 \%, 1428 \mathrm{mg} ; \mathrm{mp}: 86-87{ }^{\circ} \mathrm{C} .{ }^{1} \mathrm{H}$ NMR (400 MHz, CDCl $) \delta_{H} 7.93\left(2 \mathrm{H}, \mathrm{dd},{ }^{3} J_{\mathrm{HH}} 8.1 \mathrm{~Hz},{ }^{4} J_{\mathrm{HH}} 3.1 \mathrm{~Hz}, \mathrm{ArH}\right), 7.58-7.42(9 \mathrm{H}, \mathrm{m}$, $\mathrm{ArH}) .{ }^{13} \mathrm{C} \mathrm{NMR}\left(125 \mathrm{MHz}, \mathrm{CDCl}_{3}\right) \delta_{C} 166.3,138.1,134.9,133.7,131.8,131.4,129.0,128.5$, 127.4, 127.3, 126.5, 126.0, 125.2. HRMS (EI): [M] calcd for $\mathrm{C}_{17} \mathrm{H}_{11} \mathrm{~N}$ 229.0891; found, 229.0899 .

1-(2'-Isocyano-5'-methyl-[1, 1'-biphenyl]-4-yl)ethanone (1j). ${ }^{17}$ White solid; yield 79\%, 1485 mg; mp: $93-94^{\circ} \mathrm{C}$ (lit. $\left.{ }^{17} \mathrm{mp}: 97-99^{\circ} \mathrm{C}\right) .{ }^{1} \mathrm{H}$ NMR $\left(400 \mathrm{MHz}, \mathrm{CDCl}_{3}\right) \delta_{H} 8.06\left(2 \mathrm{H}, \mathrm{d},{ }^{3} J_{\mathrm{HH}} 8.6\right.$ $\mathrm{Hz}, \mathrm{ArH}), 7.61\left(2 \mathrm{H}, \mathrm{d},{ }^{3} J_{\mathrm{HH}} 8.3 \mathrm{~Hz}, \mathrm{ArH}\right), 7.40\left(1 \mathrm{H}, \mathrm{d},{ }^{3} J_{\mathrm{HH}} 7.9 \mathrm{~Hz}, \mathrm{ArH}\right), 7.22\left(2 \mathrm{H}, \mathrm{d},{ }^{3} J_{\mathrm{HH}} 9.1\right.$ $\mathrm{Hz}, \mathrm{ArH}), 2.66\left(3 \mathrm{H}, \mathrm{s}, \mathrm{COCH}_{3}\right), 2.43\left(3 \mathrm{H}, \mathrm{s}, \mathrm{CH}_{3}\right)$.

5-Fluoro-2-isocyanobiphenyl (1k). ${ }^{20}$ Green liquid; yield 81\%, $1276 \mathrm{mg} ;{ }^{1} \mathrm{H}$ NMR (400 MHz, $\left.\mathrm{CDCl}_{3}\right) \delta_{H} 7.50-7.44(6 \mathrm{H}, \mathrm{m}, \mathrm{ArH}), 7.2-7.1(1 \mathrm{H}, \mathrm{m}, \mathrm{ArH}), 7.1-7.0(1 \mathrm{H}, \mathrm{m}, \mathrm{ArH})$.

5-Chloro-2-isocyanobiphenyl (11). ${ }^{14}$ Pale green solid; yield $87 \%, 1482 \mathrm{mg}$; mp: $71-72{ }^{\circ} \mathrm{C}$ (lit. $\left.{ }^{14} \mathrm{mp}: 71-73{ }^{\circ} \mathrm{C}\right) .{ }^{1} \mathrm{H}$ NMR $\left(400 \mathrm{MHz}, \mathrm{CDCl}_{3}\right) \delta_{H} 7.49-7.42(7 \mathrm{H}, \mathrm{m}, \mathrm{ArH}), 7.34(1 \mathrm{H}, \mathrm{dd}$, $\left.{ }^{3} J_{\mathrm{HH}} 8.5,{ }^{4} J_{\mathrm{HH}} 2.3 \mathrm{~Hz}, \mathrm{ArH}\right)$.

2-Isocyano-3'-methoxybiphenyl (1m). ${ }^{16}$ Green solid; yield 78\%, $1304 \mathrm{mg}$; mp: $53-55{ }^{\circ} \mathrm{C}$ (lit. $\left.{ }^{16} \mathrm{mp}: 52-53{ }^{\circ} \mathrm{C}\right) .{ }^{1} \mathrm{H}$ NMR $\left(400 \mathrm{MHz}, \mathrm{CDCl}_{3}\right) \delta_{H} 7.48-7.36(5 \mathrm{H}, \mathrm{m}, \mathrm{ArH}), 7.08(1 \mathrm{H}, \mathrm{d}$, $\left.{ }^{3} J_{\mathrm{HH}} 7.6 \mathrm{~Hz}, \mathrm{ArH}\right), 7.04(1 \mathrm{H}, \mathrm{s}, \mathrm{ArH}), 6.97\left(1 \mathrm{H}, \mathrm{d},{ }^{3} J_{\mathrm{HH}} 9.1 \mathrm{~Hz}, \mathrm{ArH}\right), 3.85\left(3 \mathrm{H}, \mathrm{s}, \mathrm{OCH}_{3}\right)$.

3-(2-Isocyanophenyl)pyridine (1n). Orange solid; yield 75\%, $1086 \mathrm{mg}$; mp: $52-53{ }^{\circ} \mathrm{C} .{ }^{1} \mathrm{H}$ NMR $\left(400 \mathrm{MHz}, \mathrm{CDCl}_{3}\right) \delta_{H} 8.73\left(1 \mathrm{H}, \mathrm{d},{ }^{3} J_{\mathrm{HH}} 2.9 \mathrm{~Hz}, \mathrm{ArH}\right), 8.68\left(1 \mathrm{H}, \mathrm{dd},{ }^{3} J_{\mathrm{HH}} 4.9,{ }^{4} J_{\mathrm{HH}} 1.6 \mathrm{~Hz}\right.$, $\mathrm{ArH}), 7.91-7.87(1 \mathrm{H}, \mathrm{m}, \mathrm{ArH}), 7.56-7.48(2 \mathrm{H}, \mathrm{m}, \mathrm{ArH}), 7.48-7.40(3 \mathrm{H}, \mathrm{m}, \mathrm{ArH}) .{ }^{13} \mathrm{C} \mathrm{NMR}$ $\left(125 \mathrm{MHz}, \mathrm{CDCl}_{3}\right) \delta_{C} 167.5,149.53,149.50,136.3,135.2,132.9,130.4,129.9,129.1,128.0$, 123.2. HRMS (ESI): $[\mathrm{M}+\mathrm{H}]^{+}$calcd for $\left[\mathrm{C}_{12} \mathrm{H}_{9} \mathrm{~N}_{2}\right]^{+}$181.0760; found, 181.0760. 
General procedure for thiolation of isocyanide and intramolecular radical aromatic cyclization reaction

To a Schlenk tube were added 2-isocyanobiphenyls $1(0.30 \mathrm{mmol})$, thiols $2(0.36 \mathrm{mmol})$, xylene $(3 \mathrm{~mL})$, TBPB $(0.9 \mathrm{mmol})$, then the tube was charged with nitrogen, and was stirred at $120{ }^{\circ} \mathrm{C}$ for $5 \mathrm{~h}$. After the reaction was finished, the reaction mixture was diluted in $20 \mathrm{~mL}$ ethyl acetate, washed with a saturated solution of brine $(15 \mathrm{~mL} \times 3)$, dried with anhydrous sodium sulfate and concentrated in vacuum, and the resulting residue was purified by neutral aluminum oxide (200 - 300 mesh) or silica gel (200 - 300 mesh), chromatography to afford the product 6arylthiophenanthridines 3 .

8-Methoxy-6-(p-tolylthio)phenanthridine (3a). White solid; yield 53\%, $52 \mathrm{mg}$; mp: 155 - 156 ${ }^{\circ} \mathrm{C} .{ }^{1} \mathrm{H}$ NMR $\left(400 \mathrm{MHz}, \mathrm{CDCl}_{3}\right) \delta_{H} 8.42\left(1 \mathrm{H}, \mathrm{d},{ }^{3} J_{\mathrm{HH}} 9.0 \mathrm{~Hz}, \mathrm{ArH}\right), 8.34-8.31(1 \mathrm{H}, \mathrm{m}, \mathrm{ArH})$, $7.82-7.74(2 \mathrm{H}, \mathrm{m}, \mathrm{ArH}), 7.57-7.45(4 \mathrm{H}, \mathrm{m}, \mathrm{ArH}), 7.40\left(1 \mathrm{H}, \mathrm{dd},{ }^{3} J_{\mathrm{HH}} 9.0 \mathrm{~Hz},{ }^{4} J_{\mathrm{HH}} 2.6 \mathrm{~Hz}\right.$, $\mathrm{ArH}), 7.23\left(2 \mathrm{H}, \mathrm{d},{ }^{3} J_{\mathrm{HH}} 8.0 \mathrm{~Hz}, \mathrm{ArH}\right), 3.94\left(3 \mathrm{H}, \mathrm{s}, \mathrm{OCH}_{3}\right), 2.40\left(3 \mathrm{H}, \mathrm{s}, \mathrm{CH}_{3}\right) .{ }^{13} \mathrm{C} \mathrm{NMR}(125$ $\left.\mathrm{MHz}, \mathrm{CDCl}_{3}\right) \delta_{C} 158.8,158.2,143.3,138.6,134.9,129.8,129.3,127.6,126.9,126.9,126.7$, $126.2,124.1,123.3,121.5,121.5,105.8,55.6,21.4$. HRMS (ESI): $[\mathrm{M}+\mathrm{H}]^{+}$calcd for $\left[\mathrm{C}_{21} \mathrm{H}_{18} \mathrm{NOS}\right]^{+}$332.1104; found, 332.1105 .

8-Methoxy-6-(phenylthio)phenanthridine (3b). ${ }^{27}$ White solid; yield $58 \%, 55 \mathrm{mg}$; mp: 81 - 82 ${ }^{\circ} \mathrm{C}$ (lit. $\left.{ }^{27} \mathrm{mp}: 81-82{ }^{\circ} \mathrm{C}\right) .{ }^{1} \mathrm{H}$ NMR $\left(400 \mathrm{MHz}, \mathrm{CDCl}_{3}\right) \delta_{H} 8.49\left(1 \mathrm{H}, \mathrm{d},{ }^{3} J_{\mathrm{HH}} 9.1 \mathrm{~Hz}, \mathrm{ArH}\right), 7.40-$ $7.38(1 \mathrm{H}, \mathrm{m}, \mathrm{ArH}) 7.81-7.77(2 \mathrm{H}, \mathrm{m}, \mathrm{ArH}), 7.66-7.64(2 \mathrm{H}, \mathrm{m}, \mathrm{ArH}), 7.54-7.53(2 \mathrm{H}, \mathrm{m}$, $\mathrm{ArH}), 7.45-7.41(4 \mathrm{H}, \mathrm{m}, \mathrm{ArH}), 3.98\left(3 \mathrm{H}, \mathrm{s}, \mathrm{OCH}_{3}\right)$.

8-Methoxy-6-[(3-methoxyphenyl)thio]phenanthridine (3c). White solid; yield 45\%, $47 \mathrm{mg}$; mp: $114-115{ }^{\circ} \mathrm{C} .{ }^{1} \mathrm{H}$ NMR $\left(400 \mathrm{MHz}, \mathrm{CDCl}_{3}\right) \delta_{H} 8.50\left(1 \mathrm{H}, \mathrm{d},{ }^{3} J_{\mathrm{HH}} 9.0 \mathrm{~Hz}, \mathrm{ArH}\right), 8.40(1 \mathrm{H}, \mathrm{d}$, $\left.{ }^{3} J_{\mathrm{HH}} 9.5 \mathrm{~Hz}, \mathrm{ArH}\right), 7.86\left(1 \mathrm{H}, \mathrm{d},{ }^{3} J_{\mathrm{HH}} 9.5 \mathrm{~Hz}, \mathrm{ArH}\right), 7.78\left(1 \mathrm{H}, \mathrm{d},{ }^{3} J_{\mathrm{HH}} 2.6 \mathrm{~Hz}, \mathrm{ArH}\right), 7.58-7.53$ $(2 \mathrm{H}, \mathrm{m}, \mathrm{ArH}), 7.47\left(1 \mathrm{H}, \mathrm{dd},{ }^{3} J_{\mathrm{HH}} 9.0 \mathrm{~Hz},{ }^{4} J_{\mathrm{HH}} 2.7 \mathrm{~Hz}, \mathrm{ArH}\right), 7.32\left(1 \mathrm{H}, \mathrm{t},{ }^{3} J_{\mathrm{HH}} 7.9 \mathrm{~Hz}, \mathrm{ArH}\right), 7.24$ - $7.22(2 \mathrm{H}, \mathrm{m}, \mathrm{ArH}), 6.94\left(1 \mathrm{H}, \mathrm{d},{ }^{3} \mathrm{~J}_{\mathrm{HH}} 9.2 \mathrm{~Hz}, \mathrm{ArH}\right), 3.98\left(3 \mathrm{H}, \mathrm{s}, \mathrm{OCH}_{3}\right), 3.81\left(3 \mathrm{H}, \mathrm{s}, \mathrm{OCH}_{3}\right)$. ${ }^{13} \mathrm{C}$ NMR $\left(125 \mathrm{MHz}, \mathrm{CDCl}_{3}\right) \delta_{C} 159.8,158.8,157.6,143.3,132.1,129.6,129.4,127.7,127.1$, 126.4, 124.1, 123.5, 121.7, 121.5, 119.3, 114.6, 106.0, 55.6, 55.4. HRMS (ESI): $[\mathrm{M}+\mathrm{H}]^{+}$calcd for $\left[\mathrm{C}_{21} \mathrm{H}_{17} \mathrm{NO}_{2} \mathrm{~S}\right]^{+}$348.1053; found, 348.1054.

6-[(4-Bromophenyl)thio]-8-methoxyphenanthridine (3d). Orange solid; yield $68 \%, 80 \mathrm{mg}$; mp: $178-179{ }^{\circ} \mathrm{C} .{ }^{1} \mathrm{H}$ NMR $\left(400 \mathrm{MHz}, \mathrm{CDCl}_{3}\right) \delta_{H} 8.46\left(1 \mathrm{H}, \mathrm{d},{ }^{3} J_{\mathrm{HH}} 9.0 \mathrm{~Hz}, \mathrm{ArH}\right), 8.36(1 \mathrm{H}, \mathrm{d}$, $\left.{ }^{3} J_{\mathrm{HH}} 9.0 \mathrm{~Hz}, \mathrm{ArH}\right), 7.79\left(1 \mathrm{H}, \mathrm{d},{ }^{3} J_{\mathrm{HH}} 7.2 \mathrm{~Hz},\right), 7.70(1 \mathrm{H}, \mathrm{s}, \mathrm{ArH}), 7.56-7.50$ (6H, m, ArH), 7.44 $\left(1 \mathrm{H}, \mathrm{dd},{ }^{3} J_{\mathrm{HH}} 9.0 \mathrm{~Hz},{ }^{4} J_{\mathrm{HH}} 2.5 \mathrm{~Hz}, \mathrm{ArH}\right), 3.97\left(3 \mathrm{H}, \mathrm{s}, \mathrm{OCH}_{3}\right) .{ }^{13} \mathrm{C} \mathrm{NMR}\left(125 \mathrm{MHz}, \mathrm{CDCl}_{3}\right) \delta_{C}$ 158.9, 157.1, 143.2, 136.3, 132.1, 129.7, 129.3, 127.8, 127.0, 126.5, 124.2, 123.4, 121.7, 121.5, 105.6, 55.6. HRMS (ESI): $[\mathrm{M}+\mathrm{H}]^{+}$calcd for $\left[\mathrm{C}_{20} \mathrm{H}_{15} \mathrm{BrNOS}\right]^{+}$396.0052; found, 396.0053 .

6-[(4-Fluorophenyl)thio]-8-methoxyphenanthridine (3e). Yellow solid; yield 30\%, $30 \mathrm{mg}$; mp: $141-142{ }^{\circ} \mathrm{C} .{ }^{1} \mathrm{H}$ NMR $\left(400 \mathrm{MHz}, \mathrm{CDCl}_{3}\right) \delta_{H} 8.44\left(1 \mathrm{H}, \mathrm{d},{ }^{3} J_{\mathrm{HH}} 9.0 \mathrm{~Hz}, \mathrm{ArH}\right), 8.34(1 \mathrm{H}, \mathrm{d}$, $\left.{ }^{3} J_{\mathrm{HH}} 7.4 \mathrm{~Hz}, \operatorname{ArH}\right), 7.75-7.69(2 \mathrm{H}, \mathrm{m}, \operatorname{ArH}), 7.65-7.62(2 \mathrm{H}, \mathrm{m}, \operatorname{ArH}), 7.54-7.47$ (2H, m, $\mathrm{ArH}), 7.43\left(1 \mathrm{H}, \mathrm{dd},{ }^{3} J_{\mathrm{HH}} 9.0 \mathrm{~Hz}, 2.6 \mathrm{~Hz}, \mathrm{ArH}\right), 7.14\left(2 \mathrm{H}, \mathrm{t},{ }^{3} J_{\mathrm{HH}} 8.7 \mathrm{~Hz}, \mathrm{ArH}\right), 3.97(3 \mathrm{H}, \mathrm{s}$, $\left.\mathrm{OCH}_{3}\right) .{ }^{13} \mathrm{C}$ NMR $\left(125 \mathrm{MHz}, \mathrm{CDCl}_{3}\right) \delta_{\mathrm{C}} 164.5,162.0,158.9,157.8,143.2,137.4,137.3,129.2$, 
127.7, 126.3, 124.2, 121.6, 121.5, 116.2, 116.0, 105.5, 55.6. HRMS (ESI): $[\mathrm{M}+\mathrm{H}]^{+}$calcd for $\left[\mathrm{C}_{20} \mathrm{H}_{15} \mathrm{FNOS}\right]^{+}$336.0853; found, 336.0855.

6-(Ethylthio)-8-methoxyphenanthridine (3f). Yellow liquid; yield 45\%, $36 \mathrm{mg} .{ }^{1} \mathrm{H}$ NMR (400 $\left.\mathrm{MHz}, \mathrm{CDCl}_{3}\right) \delta_{H} 8.44\left(1 \mathrm{H}, \mathrm{d},{ }^{3} J_{\mathrm{HH}} 9.1 \mathrm{~Hz},\right), 8.38-8.36(1 \mathrm{H}, \mathrm{m}, \mathrm{ArH}), 7.99\left(1 \mathrm{H}, \mathrm{d},{ }^{3} J_{\mathrm{HH}} 8.1 \mathrm{~Hz}\right.$, ArH), $7.62-7.58(2 \mathrm{H}, \mathrm{m}, \mathrm{ArH}), 7.53-7.51(1 \mathrm{H}, \mathrm{m}, \mathrm{ArH}), 7.41\left(1 \mathrm{H}, \mathrm{d},{ }^{3} \mathrm{~J}_{\mathrm{HH}} 9.0 \mathrm{~Hz}, \mathrm{ArH}\right), 3.97$ $\left(3 \mathrm{H}, \mathrm{s}, \mathrm{OCH}_{3}\right), 3.49\left(2 \mathrm{H}, \mathrm{q},{ }^{3} \mathrm{~J}_{\mathrm{HH}} 7.4 \mathrm{~Hz}, \mathrm{CH}_{2} \mathrm{CH}_{3}\right), 1.50\left(3 \mathrm{H}, \mathrm{t},{ }^{3} \mathrm{~J}_{\mathrm{HH}} 7.4 \mathrm{~Hz}, \mathrm{CH}_{2} \mathrm{CH}_{3}\right) .{ }^{13} \mathrm{C} \mathrm{NMR}$ $\left(125 \mathrm{MHz}, \mathrm{CDCl}_{3}\right) \delta_{C} 157.7,157.3,142.4,127.6,126.6,125.9,125.3,124.6,123.0,121.8$, 120.6, 120.2, 104.4, 54.5, 23.2, 13.6. HRMS (ESI): $[\mathrm{M}+\mathrm{H}]^{+}$calcd for $\left[\mathrm{C}_{16} \mathrm{H}_{16} \mathrm{NOS}\right]^{+} 270.0947$; found, 270.0955 .

6-(Phenylthio)phenanthridine (3g). ${ }^{27}$ Pale yellow solid; yield 53\%, $45 \mathrm{mg}$; mp: $73-75{ }^{\circ} \mathrm{C}$ (lit. $\left.{ }^{27} \mathrm{mp}: 71-72{ }^{\circ} \mathrm{C}\right) .{ }^{1} \mathrm{H} \mathrm{NMR}\left(400 \mathrm{MHz}, \mathrm{CDCl}_{3}\right) \delta_{H} 8.53\left(1 \mathrm{H}, \mathrm{d},{ }^{3} J_{\mathrm{HH}} 8.2 \mathrm{~Hz}, \mathrm{ArH}\right), 8.44(1 \mathrm{H}$, d, $\left.{ }^{3} J_{\mathrm{HH}} 8.6 \mathrm{~Hz}, \mathrm{ArH}\right), 8.41(1 \mathrm{H}, \mathrm{s}, \mathrm{ArH}), 7.81-7.77(2 \mathrm{H}, \mathrm{m}, \mathrm{ArH}), 7.67-7.63$ (3H, m, ArH), $7.58-7.48$ (2H, m, ArH), 7.45 - 7.37 (3H, m, ArH).

1-(6-(Phenylthio)phenanthridin-8-yl)ethanone (3h). ${ }^{27}$ White solid; yield 41\%, $41 \mathrm{mg}$; mp: 107 - $108{ }^{\circ} \mathrm{C}$ (lit. $\left.{ }^{27} \mathrm{mp}: 107-108{ }^{\circ} \mathrm{C}\right) .{ }^{1} \mathrm{H}$ NMR $\left(400 \mathrm{MHz}, \mathrm{CDCl}_{3}\right) \delta_{H} 8.99(1 \mathrm{H}, \mathrm{s}, \mathrm{ArH}), 8.59(1 \mathrm{H}$, $\left.\mathrm{d},{ }^{3} J_{\mathrm{HH}} 8.6 \mathrm{~Hz}, \mathrm{ArH}\right), 8.44\left(1 \mathrm{H}, \mathrm{d},{ }^{3} J_{\mathrm{HH}} 8.1 \mathrm{~Hz}, \mathrm{ArH}\right), 8.36\left(1 \mathrm{H}, \mathrm{d},{ }^{3} J_{\mathrm{HH}} 8.8 \mathrm{~Hz}, \mathrm{ArH}\right), 7.79(1 \mathrm{H}$, d, $\left.{ }^{3} J_{\mathrm{HH}} 8.0 \mathrm{~Hz}, \mathrm{ArH}\right), 7.69-7.64(3 \mathrm{H}, \mathrm{m}, \mathrm{ArH}), 7.57-7.55$ (1H, t, ArH), $7.46-7.44$ (3H, m, $\mathrm{ArH}), 2.76\left(3 \mathrm{H}, \mathrm{s}, \mathrm{CH}_{3}\right)$.

Methyl 6-(phenylthio)phenanthridine-8-carboxylate (3i). Yellow solid; yield 50\%, $52 \mathrm{mg}$; mp: $129-130{ }^{\circ} \mathrm{C} .{ }^{1} \mathrm{H}$ NMR $\left(400 \mathrm{MHz}, \mathrm{CDCl}_{3}\right) \delta_{H} 9.13(1 \mathrm{H}, \mathrm{s}, \mathrm{ArH}), 8.56\left(1 \mathrm{H}, \mathrm{d},{ }^{3} J_{\mathrm{HH}} 8.6 \mathrm{~Hz}\right.$, ArH), $8.40\left(2 \mathrm{H}, \mathrm{dd},{ }^{3} J_{\mathrm{HH}} 11.5 \mathrm{~Hz},{ }^{4} J_{\mathrm{HH}} 4.1 \mathrm{~Hz}, \mathrm{ArH}\right), 7.75\left(1 \mathrm{H}, \mathrm{dd},{ }^{3} J_{\mathrm{HH}} 8.1,{ }^{4} J_{\mathrm{HH}} 1.1 \mathrm{~Hz}, \mathrm{ArH}\right)$, $7.70-7.59$ (2H, m, ArH), $7.63-7.59$ (1H, t, ArH), $7.55-7.51(1 \mathrm{H}, \mathrm{t}, \mathrm{ArH}), 7.48-7.43(3 \mathrm{H}, \mathrm{m}$, $\mathrm{ArH}), 4.02\left(3 \mathrm{H}, \mathrm{s}, \mathrm{CH}_{3}\right) .{ }^{13} \mathrm{C} \mathrm{NMR}\left(125 \mathrm{MHz}, \mathrm{CDCl}_{3}\right) \delta_{\mathrm{C}} 166.3,159.7,144.8,135.7,135.4$, 130.7, 129.8, 129.7, 129.4, 129.0, 128.9, 128.8, 127.8, 126.4, 124.8, 122.8, 122.6, 122.4, 52.5 . HRMS (ESI): $[\mathrm{M}+\mathrm{H}]^{+}$calcd for $\left[\mathrm{C}_{21} \mathrm{H}_{16} \mathrm{NO}_{2} \mathrm{~S}\right]^{+} 346.0896$; found, 346.0888.

8-Chloro-6-(phenylthio)phenanthridine (3j). ${ }^{28}$ White solid; yield 47\%, $45 \mathrm{mg}$; mp: 112 - 114 ${ }^{\circ} \mathrm{C}$ (lit. $\left.{ }^{28} \mathrm{mp}: 115-116{ }^{\circ} \mathrm{C}\right) .{ }^{1} \mathrm{H}$ NMR $\left(400 \mathrm{MHz}, \mathrm{CDCl}_{3}\right) \delta_{H} 8.43(1 \mathrm{H}, \mathrm{s}, \mathrm{ArH}), 8.40(1 \mathrm{H}, \mathrm{s}$, ArH), $8.32\left(1 \mathrm{H}, \mathrm{dd},{ }^{3} J_{\mathrm{HH}} 8.0 \mathrm{~Hz},{ }^{4} J_{\mathrm{HH}} 1.4 \mathrm{~Hz}, \mathrm{ArH}\right), 7.76-7.70(2 \mathrm{H}, \mathrm{m}, \mathrm{ArH}), 7.66-7.64(2 \mathrm{H}$, m, ArH), 7.58 - 7.54 (1H, m, ArH), $7.52-7.48$ (1H, m, ArH), $7.45-7.41$ (3H, m, ArH).

8-Fluoro-6-(phenylthio)phenanthridine (3k). ${ }^{27}$ White solid; yield 52\%, $47 \mathrm{mg}$; mp: $101-102$ ${ }^{\circ} \mathrm{C}$ (lit. $\left.{ }^{27} \mathrm{mp}: 97-98{ }^{\circ} \mathrm{C}\right) .{ }^{1} \mathrm{H}$ NMR $\left(400 \mathrm{MHz}, \mathrm{CDCl}_{3}\right) \delta_{H} 8.50\left(1 \mathrm{H}, \mathrm{dd},{ }^{3} J_{\mathrm{HH}} 9.1 \mathrm{~Hz},{ }^{4} J_{\mathrm{HH}} 5.3\right.$ $\mathrm{Hz}, \mathrm{ArH}), 8.33\left(1 \mathrm{H}, \mathrm{d},{ }^{3} J_{\mathrm{HH}} 8.0 \mathrm{~Hz}, \mathrm{ArH}\right), 8.07\left(1 \mathrm{H}, \mathrm{dd},{ }^{3} J_{\mathrm{HH}} 9.7,2.6 \mathrm{~Hz}, \mathrm{ArH}\right), 7.77(1 \mathrm{H}, \mathrm{dd}$, $\left.{ }^{3} J_{\mathrm{HH}} 8.0 \mathrm{~Hz},{ }^{4} J_{\mathrm{HH}} 1.3 \mathrm{~Hz}, \mathrm{ArH}\right), 7.66-7.63(2 \mathrm{H}, \mathrm{m}, \mathrm{ArH}), 7.57-7.49$ (3H, m, ArH), $7.45-7.40$ $(3 \mathrm{H}, \mathrm{m}, \mathrm{ArH})$.

6-(Phenylthio)phenanthridine-8-carbonitrile (3I). Yellow solid; yield 45\%, $42 \mathrm{mg}$; mp: 182 $183{ }^{\circ} \mathrm{C} .{ }^{1} \mathrm{H}$ NMR $\left(400 \mathrm{MHz}, \mathrm{CDCl}_{3}\right) \delta_{H} 8.75(1 \mathrm{H}, \mathrm{s}, \mathrm{ArH}), 8.57\left(1 \mathrm{H}, \mathrm{d},{ }^{3} J_{\mathrm{HH}} 8.6 \mathrm{~Hz}, \mathrm{ArH}\right), 8.38$ $\left(1 \mathrm{H}, \mathrm{d},{ }^{3} J_{\mathrm{HH}} 8.0 \mathrm{~Hz}, \mathrm{ArH}\right), 7.95\left(1 \mathrm{H}, \mathrm{d},{ }^{3} J_{\mathrm{HH}} 8.6 \mathrm{~Hz}, \mathrm{ArH}\right), 7.76\left(1 \mathrm{H}, \mathrm{d},{ }^{3} J_{\mathrm{HH}} 8.0 \mathrm{~Hz}, \mathrm{ArH}\right) 7.67-$ $7.63(3 \mathrm{H}, \mathrm{m}, \mathrm{ArH}), 7.59-7.55$ (1H, m, ArH), 7.47 - 7.46 (3H, m, ArH). ${ }^{13} \mathrm{C} \mathrm{NMR}(125 \mathrm{MHz}$, $\left.\mathrm{CDCl}_{3}\right) \delta_{C} 158.6,145.0,135.4,135.3,132.1,130.9,130.5,129.6,129.1,126.9,124.8,123.7$, 
122.5, 121.8, 118.4, 110.9. HRMS (ESI): $[\mathrm{M}+\mathrm{H}]^{+}$calcd for $\left[\mathrm{C}_{20} \mathrm{H}_{13} \mathrm{~N}_{2} \mathrm{~S}\right]^{+}$313.0794; found, 313.0788 .

8-(t-Butyl)-6-(phenylthio)phenanthridine (3m). Orange liquid; yield 60\%, $62 \mathrm{mg} .{ }^{1} \mathrm{H}$ NMR $\left(400 \mathrm{MHz}, \mathrm{CDCl}_{3}\right) \delta_{H} 8.47\left(1 \mathrm{H}, \mathrm{d},{ }^{3} J_{\mathrm{HH}} 8.7 \mathrm{~Hz}, \mathrm{ArH}\right), 8.41-8.39(2 \mathrm{H}, \mathrm{m}, \mathrm{ArH}), 7.88(1 \mathrm{H}, \mathrm{dd}$, $\left.{ }^{3} J_{\mathrm{HH}} 8.7 \mathrm{~Hz},{ }^{4} J_{\mathrm{HH}} 1.9 \mathrm{~Hz}, \mathrm{ArH}\right), 7.79\left(1 \mathrm{H}, \mathrm{dd},{ }^{3} J_{\mathrm{HH}} 8.1 \mathrm{~Hz},{ }^{4} J_{\mathrm{HH}} 1.1 \mathrm{~Hz}, \mathrm{ArH}\right), 7.66\left(2 \mathrm{H}, \mathrm{dd},{ }^{3} J_{\mathrm{HH}}\right.$ $7.8 \mathrm{~Hz}, 1.5 \mathrm{~Hz}, \mathrm{ArH}), 7.55-7.50$ (2H, m, ArH), $7.43-7.37$ (3H, m, ArH), 1.44 (9H, s, $t$-Bu). ${ }^{13} \mathrm{C} \mathrm{NMR}\left(125 \mathrm{MHz}, \mathrm{CDCl}_{3}\right) \delta_{C} 159.1,150.8,143.9,134.8,130.9,130.5,129.4,129.3,129.0$, 128.4, 128.3, 126.2, 125.4, 123.3, 122.3, 121.9, 121.6, 35.2, 31.4. HRMS (ESI): $[\mathrm{M}+\mathrm{H}]^{+}$calcd for $\left[\mathrm{C}_{23} \mathrm{H}_{22} \mathrm{NS}\right]^{+}$344.1467; found, 344.1468.

8-(t-Butyl)-6-(p-tolylthio)phenanthridine (3n). Yellow liquid; yield 60\%, $64 \mathrm{mg} .{ }^{1} \mathrm{H}$ NMR $\left(400 \mathrm{MHz}, \mathrm{CDCl}_{3}\right) \delta_{H} 8.49\left(1 \mathrm{H}, \mathrm{d},{ }^{3} J_{\mathrm{HH}} 8.7 \mathrm{~Hz}, \mathrm{ArH}\right), 8.43-8.40(2 \mathrm{H}, \mathrm{m}, \mathrm{ArH}), 7.90(1 \mathrm{H}, \mathrm{dd}$, $\left.{ }^{3} J_{\mathrm{HH}} 8.7 \mathrm{~Hz},{ }^{4} J_{\mathrm{HH}} 1.9 \mathrm{~Hz}, \mathrm{ArH}\right), 7.79\left(1 \mathrm{H}, \mathrm{dd},{ }^{3} J_{\mathrm{HH}} 8.1 \mathrm{~Hz},{ }^{4} J_{\mathrm{HH}} 1.2 \mathrm{~Hz}, \mathrm{ArH}\right), 7.56-7.49(4 \mathrm{H}, \mathrm{m}$, ArH), $7.24\left(2 \mathrm{H}, \mathrm{d},{ }^{3} J_{\mathrm{HH}} 7.9 \mathrm{~Hz}, \mathrm{ArH}\right), 2.41\left(3 \mathrm{H}, \mathrm{s}, \mathrm{CH}_{3}\right), 1.46(9 \mathrm{H}, \mathrm{s}, t-\mathrm{Bu}) .{ }^{13} \mathrm{C} \mathrm{NMR}(125 \mathrm{MHz}$, $\left.\mathrm{CDCl}_{3}\right) \delta_{C} 159.5,150.7,143.9,138.5,135.0,130.4,129.7,129.3,129.2,128.2,126.9,126.0$, $125.3,123.3,122.2,121.8,121.5,35.2,31.3,21.4$. HRMS (ESI): $[\mathrm{M}+\mathrm{H}]^{+}$calcd for $\left[\mathrm{C}_{24} \mathrm{H}_{24} \mathrm{NS}\right]^{+}$358.1624; found, 358.1627.

8-Chloro-6-(p-tolylthio)phenanthridine (3o). Pale green solid; yield 55\%, $55 \mathrm{mg}$; mp: 141 $142{ }^{\circ} \mathrm{C} .{ }^{1} \mathrm{H}$ NMR $\left(400 \mathrm{MHz}, \mathrm{CDCl}_{3}\right) \delta_{H} 8.48\left(1 \mathrm{H}, \mathrm{d},{ }^{3} J_{\mathrm{HH}} 8.8 \mathrm{~Hz}, \mathrm{ArH}\right), 8.44(1 \mathrm{H}, \mathrm{s}, \mathrm{ArH}), 8.38$ $\left(1 \mathrm{H}, \mathrm{d},{ }^{3} J_{\mathrm{HH}} 8.0 \mathrm{~Hz}, \mathrm{ArH}\right), 7.77\left(2 \mathrm{H}, \mathrm{t},{ }^{3} J_{\mathrm{HH}} 7.0 \mathrm{~Hz}, \mathrm{ArH}\right), 7.60-7.51(4 \mathrm{H}, \mathrm{m}, \mathrm{ArH}), 7.26(2 \mathrm{H}, \mathrm{d}$, $\left.{ }^{3} J_{\mathrm{HH}} 8.0 \mathrm{~Hz}, \mathrm{ArH}\right), 2.43(3 \mathrm{H}, \mathrm{s}, \mathrm{ArH}) .{ }^{13} \mathrm{C} \mathrm{NMR}\left(125 \mathrm{MHz}, \mathrm{CDCl}_{3}\right) \delta_{\mathrm{C}} 158.3,144.0,138.9$, 135.3, 133.4, 131.4, 131.0, 129.8, 129.4, 129.0, 126.4, 126.3, 126.0, 125.1, 124.2, 122.5, 121.8, 21.4. HRMS (ESI): $[\mathrm{M}+\mathrm{H}]^{+}$calcd for $\left[\mathrm{C}_{20} \mathrm{H}_{15} \mathrm{NSCl}\right]^{+} 336.0608$; found, 336.0609 .

6-(p-Tolylthio)phenanthridine (3p) ${ }^{27}$ White solid; yield 55\%, $50 \mathrm{mg}$; mp: $106-107{ }^{\circ} \mathrm{C}$ (lit. ${ }^{27}$ mp: $\left.106-107^{\circ} \mathrm{C}\right) .{ }^{1} \mathrm{H}$ NMR $\left(400 \mathrm{MHz}, \mathrm{CDCl}_{3}\right) \delta_{H} 8.54\left(1 \mathrm{H}, \mathrm{d},{ }^{3} J_{\mathrm{HH}} 8.2 \mathrm{~Hz}, \mathrm{ArH}\right), 8.43(2 \mathrm{H}, \mathrm{dd}$, $\left.{ }^{3} J_{\mathrm{HH}} 12.6 \mathrm{~Hz},{ }^{4} J_{\mathrm{HH}} 4.6 \mathrm{~Hz}, \mathrm{ArH}\right), 7.82-7.78(2 \mathrm{H}, \mathrm{m}, \mathrm{ArH}), 7.68-7.64$ (1H, m, ArH), 7.58 $7.50(4 \mathrm{H}, \mathrm{m}, \mathrm{ArH}), 7.25-7.22(2 \mathrm{H}, \mathrm{m}, \mathrm{ArH}), 2.41\left(3 \mathrm{H}, \mathrm{s}, \mathrm{CH}_{3}\right)$.

6-[(4-Bromophenyl)thio]benzo $[\boldsymbol{k}]$ phenanthridine (3q). Pale yellow solid; yield 55\%, $68 \mathrm{mg}$; mp: $179-181{ }^{\circ} \mathrm{C} .{ }^{1} \mathrm{H}$ NMR $\left(400 \mathrm{MHz}, \mathrm{CDCl}_{3}\right) \delta_{H} 9.12-9.10(1 \mathrm{H}, \mathrm{m}, \mathrm{ArH}), 8.92\left(1 \mathrm{H}, \mathrm{d},{ }^{3} J_{\mathrm{HH}}\right.$ $8.3 \mathrm{~Hz}, \mathrm{ArH}), 8.34\left(1 \mathrm{H}, \mathrm{d},{ }^{3} J_{\mathrm{HH}} 8.8 \mathrm{~Hz}, \mathrm{ArH}\right), 8.07-8.04(1 \mathrm{H}, \mathrm{m}, \mathrm{ArH}), 8.00\left(1 \mathrm{H}, \mathrm{d},{ }^{3} J_{\mathrm{HH}} 8.8\right.$ $\mathrm{Hz}, \mathrm{ArH}), 7.93\left(1 \mathrm{H}, \mathrm{d},{ }^{3} J_{\mathrm{HH}} 8.0 \mathrm{~Hz}, \mathrm{ArH}\right), 7.75-7.72(2 \mathrm{H}, \mathrm{m}, \mathrm{ArH}), 7.68-7.52(6 \mathrm{H}, \mathrm{m}, \mathrm{ArH})$. ${ }^{13} \mathrm{C} \mathrm{NMR}\left(125 \mathrm{MHz}, \mathrm{CDCl}_{3}\right) \delta_{C} 157.7,145.9,136.4,135.0,132.0,131.8,123.0,129.3,129.1$, $128.7,128.7,128.5,128.4,128.0,127.1,127.0,126.0,124.1,123.6,122.9,121.8$. HRMS (ESI): $[\mathrm{M}+\mathrm{H}]^{+}$calcd for $\left[\mathrm{C}_{23} \mathrm{H}_{15} \mathrm{BrNS}\right]^{+}$416.0103; found, 415.9980 .

1-\{6-[(4-Bromophenyl)thio]-2-methylphenanthridin-8-yl\}ethanone (3r). Orange oil; yield 40\%, $50 \mathrm{mg} .{ }^{1} \mathrm{H}$ NMR $\left(400 \mathrm{MHz}, \mathrm{CDCl}_{3}\right) \delta_{H} 8.92(1 \mathrm{H}, \mathrm{s}, \mathrm{ArH}), 8.56\left(1 \mathrm{H}, \mathrm{d},{ }^{3} J_{\mathrm{HH}} 8.5 \mathrm{~Hz}, \mathrm{ArH}\right)$, $8.33\left(1 \mathrm{H}, \mathrm{d},{ }^{3} J_{\mathrm{HH}} 8.4 \mathrm{~Hz}, \mathrm{ArH}\right), 8.21(1 \mathrm{H}, \mathrm{s}, \mathrm{ArH}), 7.70\left(1 \mathrm{H}, \mathrm{d},{ }^{3} J_{\mathrm{HH}} 8.2 \mathrm{~Hz}, \operatorname{ArH}\right), 7.58-7.47$ $(5 \mathrm{H}, \mathrm{m}, \mathrm{ArH}), 2.76\left(3 \mathrm{H}, \mathrm{s}, \mathrm{COCH}_{3}\right), 2.57\left(3 \mathrm{H}, \mathrm{s}, \mathrm{ArCH}_{3}\right) .{ }^{13} \mathrm{C} \mathrm{NMR}\left(125 \mathrm{MHz}, \mathrm{CDCl}_{3}\right) \delta_{C}$ 197.0, 157.6, 143.2, 136.8, 136.5, 135.6, 135.4, 132.1, 131.7, 129.2, 129.1, 126.7, 124.8, 123.2, 123.0, 122.31, 122.27, 26.7, 21.9. HRMS (ESI): $[\mathrm{M}+\mathrm{H}]^{+}$calcd for $\left[\mathrm{C}_{22} \mathrm{H}_{17} \mathrm{NOS}\right]^{+} 422.0209$; found, 422.0210 . 
2-Fluoro-6-(naphthalen-2-ylthio)phenanthridine (3s). White solid; yield 41\%, $44 \mathrm{mg}$; mp: $169-170{ }^{\circ} \mathrm{C} .{ }^{1} \mathrm{H}$ NMR $\left(400 \mathrm{MHz}, \mathrm{CDCl}_{3}\right) \delta_{H} 8.50\left(1 \mathrm{H}, \mathrm{d},{ }^{3} J_{\mathrm{HH}} 8.0 \mathrm{~Hz}, \mathrm{ArH}\right), 8.45\left(1 \mathrm{H}, \mathrm{d},{ }^{3} J_{\mathrm{HH}}\right.$ $8.4 \mathrm{~Hz}, \mathrm{ArH}), 8.18(1 \mathrm{H}, \mathrm{s}, \mathrm{ArH}), 8.05\left(1 \mathrm{H}, \mathrm{dd},{ }^{3} J_{\mathrm{HH}} 10.0 \mathrm{~Hz},{ }^{4} J_{\mathrm{HH}} 2.7 \mathrm{~Hz}, \mathrm{ArH}\right), 7.89-7.83(4 \mathrm{H}$, $\mathrm{m}, \mathrm{ArH}), 7.74-7.68(3 \mathrm{H}, \mathrm{m}, \mathrm{ArH}), 7.56-7.51(2 \mathrm{H}, \mathrm{m}, \mathrm{ArH}), 7.30-7.25(1 \mathrm{H}, \mathrm{m}, \mathrm{ArH}) .{ }^{13} \mathrm{C}$ NMR $\left(125 \mathrm{MHz}, \mathrm{CDCl}_{3}\right) \delta_{C} 161.8,159.8,158.5,141.0,140.9,134.0,133.8,133.1,132.1,131.5$, $131.4,131.0,128.2,128.2,127.9,127.8,127.7,126.7,126.3,125.9,125.5,124.5,124.4,122.6$, 117.4, 117.2, 107.1, 107.0. HRMS (ESI): $[\mathrm{M}+\mathrm{H}]^{+}$calcd for $\left[\mathrm{C}_{23} \mathrm{H}_{15} \mathrm{FNS}\right]^{+}$356.0904; found, 356.0906.

6-[(4-Bromophenyl)thio]-2-chlorophenanthridine (3t). Yellow solid; yield 60\%, $72 \mathrm{mg}$; mp: $142-143{ }^{\circ} \mathrm{C} .{ }^{1} \mathrm{H}$ NMR $\left(400 \mathrm{MHz}, \mathrm{CDCl}_{3}\right) \delta_{H} 8.51\left(1 \mathrm{H}, \mathrm{d},{ }^{3} J_{\mathrm{HH}} 8.2 \mathrm{~Hz}, \mathrm{ArH}\right), 8.42-8.41(2 \mathrm{H}$, m, ArH), $7.88\left(1 \mathrm{H}, \mathrm{t},{ }^{3} J_{\mathrm{HH}} 7.7 \mathrm{~Hz}, \mathrm{ArH}\right), 7.77-7.70\left(2 \mathrm{H}, \mathrm{dd},{ }^{3} J_{\mathrm{HH}} 17.6 \mathrm{~Hz},{ }^{4} J_{\mathrm{HH}} 7.9 \mathrm{~Hz}, \mathrm{ArH}\right)$, $7.60-7.52$ (5H, m, ArH). ${ }^{13} \mathrm{C}$ NMR $\left(125 \mathrm{MHz}, \mathrm{CDCl}_{3}\right) \delta_{C} 158.9,142.3,136.9,132.1,132.0$, $131.5,131.3,130.7,129.2,128.8,128.2,125.5,125.2,124.2,123.3,122.5,121.7$. HRMS (ESI): $[\mathrm{M}+\mathrm{H}]^{+}$calcd for $\left[\mathrm{C}_{19} \mathrm{H}_{12} \mathrm{BrClNS}\right]^{+}$399.9557; found, 399.9560 .

7-Methoxy-6-(phenylthio)phenanthridine (3u) and 9-methoxy-6-(phenylthio) phenanthri-

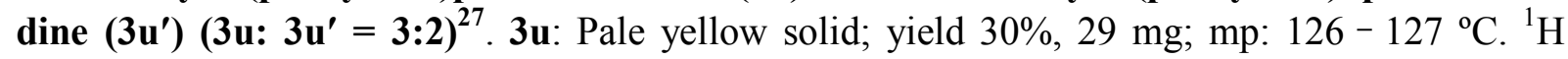
NMR (400 MHz, $\left.\mathrm{CDCl}_{3}\right) \delta_{H} 8.40\left(2 \mathrm{H}, \mathrm{d},{ }^{3} J_{\mathrm{HH}} 9.0 \mathrm{~Hz}, \mathrm{ArH}\right), 7.90\left(1 \mathrm{H}, \mathrm{d},{ }^{3} J_{\mathrm{HH}} 2.5 \mathrm{~Hz}, \mathrm{ArH}\right), 7.79$ $\left(1 \mathrm{H}, \mathrm{dd},{ }^{3} J_{\mathrm{HH}} 8.1 \mathrm{~Hz},{ }^{4} J_{\mathrm{HH}} 1.2 \mathrm{~Hz}, \mathrm{ArH}\right), 7.65-7.63(2 \mathrm{H}, \mathrm{m}, \mathrm{ArH}), 7.59-7.57(1 \mathrm{H}, \mathrm{m}, \mathrm{ArH})$, $7.54-7.52(1 \mathrm{H}, \mathrm{m}, \mathrm{ArH}), 7.44-7.39(3 \mathrm{H}, \mathrm{m}, \mathrm{ArH}), 7.28\left(1 \mathrm{H}, \mathrm{dd},{ }^{3} J_{\mathrm{HH}} 9.0 \mathrm{~Hz},{ }^{4} J_{\mathrm{HH}} 2.5 \mathrm{~Hz}\right.$, $\mathrm{ArH}), 4.04$ (3H, s, $\left.\mathrm{OCH}_{3}\right)$. 3u': Pale yellow solid; yield 20\%, $20 \mathrm{mg}$; mp: $116-117^{\circ} \mathrm{C} .{ }^{1} \mathrm{H}$ NMR $\left(400 \mathrm{MHz}, \mathrm{CDCl}_{3}\right) \delta_{H} 8.37\left(1 \mathrm{H}, \mathrm{d},{ }^{3} J_{\mathrm{HH}} 8.0 \mathrm{~Hz}, 1 \mathrm{H}\right), 8.17\left(1 \mathrm{H}, \mathrm{d},{ }^{3} J_{\mathrm{HH}} 8.0 \mathrm{~Hz}, \mathrm{ArH}\right), 7.73(1 \mathrm{H}, \mathrm{t}$, $\left.{ }^{3} J_{\mathrm{HH}} 8.1 \mathrm{~Hz}, \mathrm{ArH}\right), 7.66-7.64(2 \mathrm{H}, \mathrm{m}, \mathrm{ArH}), 7.52-7.51(2 \mathrm{H}, \mathrm{m}, \mathrm{ArH}), 7.49-7.42(4 \mathrm{H}, \mathrm{m}$, $\mathrm{ArH}), 7.13\left(1 \mathrm{H}, \mathrm{d},{ }^{3} J_{\mathrm{HH}} 7.9 \mathrm{~Hz}, \mathrm{ArH}\right), 4.14\left(3 \mathrm{H}, \mathrm{s}, \mathrm{OCH}_{3}\right)$.

5-(Phenylthio)benzo[f][1,7]naphthyridine $(3 v)$ and 5-(phenylthio)benzo[c] $[2,6]$ naphthyridine $\left(3 \mathbf{v}^{\prime}\right)\left(3 \mathbf{v}: 3 \mathbf{v}^{\prime}=3: 2\right)$. 3v: Yellow solid; yield 47\%, $41 \mathrm{mg} ; \mathrm{mp}: 109-110{ }^{\circ} \mathrm{C} .{ }^{1} \mathrm{H} \mathrm{NMR}$ $\left(400 \mathrm{MHz}, \mathrm{CDCl}_{3}\right) \delta_{H} 9.04\left(1 \mathrm{H}, \mathrm{dd},{ }^{3} J_{\mathrm{HH}} 4.4 \mathrm{~Hz},{ }^{4} J_{\mathrm{HH}} 1.5 \mathrm{~Hz}, \mathrm{ArH}\right), 8.83\left(1 \mathrm{H}, \mathrm{dd},{ }^{3} J_{\mathrm{HH}} 8.4 \mathrm{~Hz}\right.$, $\left.{ }^{4} J_{\mathrm{HH}} 1.5 \mathrm{~Hz}, \mathrm{ArH}\right), 8.37\left(1 \mathrm{H}, \mathrm{dd},{ }^{3} J_{\mathrm{HH}} 8.0 \mathrm{~Hz},{ }^{4} J_{\mathrm{HH}} 1.2 \mathrm{~Hz}, \mathrm{ArH}\right), 7.78-7.75(4 \mathrm{H}, \mathrm{m}, \mathrm{ArH}), 7.63$ - 7.59 (1H, m, ArH), $7.56-7.47$ (4H, m, ArH). $\left.{ }^{13} \mathrm{C} \mathrm{NMR} \mathrm{(125} \mathrm{MHz,} \mathrm{CDCl}_{3}\right) \delta_{C} 162.2,149.6$, $144.3,140.2,135.9,130.7,129.5,129.4,128.8,128.8,126.2,122.1,122.0$. HRMS (ESI): $[\mathrm{M}+$ $\mathrm{H}]^{+}$calcd for $\left[\mathrm{C}_{18} \mathrm{H}_{13} \mathrm{~N}_{2} \mathrm{~S}\right]^{+}$289.0794; found, 289.0796. 3v': Yellow solid; yield 32\%, $28 \mathrm{mg}$; mp: $108-109{ }^{\circ} \mathrm{C} .{ }^{1} \mathrm{H}$ NMR $\left(400 \mathrm{MHz}, \mathrm{CDCl}_{3}\right) \delta_{H} 9.99(1 \mathrm{H}, \mathrm{s}, \mathrm{ArH}), 8.87\left(1 \mathrm{H}, \mathrm{d},{ }^{3} J_{\mathrm{HH}} 5.6 \mathrm{~Hz}\right.$, ArH), $8.58-8.49(1 \mathrm{H}, \mathrm{m}, \mathrm{ArH}), 8.15\left(1 \mathrm{H}, \mathrm{d},{ }^{3} J_{\mathrm{HH}} 5.6 \mathrm{~Hz}, \mathrm{ArH}\right), 7.82-7.77(1 \mathrm{H}, \mathrm{m}, \mathrm{ArH}), 7.68$ - 7.58 (4H, m, ArH), $7.48-7.44(3 \mathrm{H}, \mathrm{m}, \mathrm{ArH}) .{ }^{13} \mathrm{C} \mathrm{NMR}\left(125 \mathrm{MHz}, \mathrm{CDCl}_{3}\right) \delta_{\mathrm{C}} 158.3,147.1$, $146.5,144.6,135.3,129.6,129.5,129.1,129.0,128.9$, 128.6, 127.2, 126.5, 121.4, 121.1, 117.6. HRMS (ESI): $[\mathrm{M}+\mathrm{H}]^{+}$calcd for $\left[\mathrm{C}_{18} \mathrm{H}_{13} \mathrm{~N}_{2} \mathrm{~S}\right]^{+}$289.0794; found, 289.0796.

\section{Acknowledgements}

The authors gratefully acknowledge the financial support of the National Natural Science Foundation of China (No. 21576041) and the program for Changjiang Scholars and Innovative 
Research Team in University (No. IRT13008). We also thank Prof. Baomin Wang for valuable discussions.

\section{References}

1. Lippard, S. J. Proc. Natl. Acad. Sci. USA 2012, 109, 11987. http://dx.doi.org/10.1073/pnas. 1207670109

2. Nakanishi, T.; Masuda, A.; Suwa, M.; Akiyama, Y.; Hoshino-Abe, N.; Suzuki, M. Bioorg. Med. Chem. Lett. 2000, 10, 2321. http://dx.doi.org/10.1016/S0960-894X(00)00467-4

3. Fang, S. D.; Wang, L. K.; Hecht, S. M. J. Org. Chem.1993, 58, 5025. http://dx.doi.org/10.1021/j000071a001

4. Nakanishi, T.; Suzuki, M.; Saimoto, A.; Kabasawa, T. J. Nat. Prod. 1999, 62, 864. http://dx.doi.org/10.1021/np990005d

5. Nakanishi, T.; Suzuki, M. J. Nat. Prod. 1998, 61, 1263. http://dx.doi.org/10.1021/np980193s

6. Chen, H.; Long, H.; Cui, X.; Zhou, J.; Xu, M.; Yuan, G. J. Am. Chem. Soc. 2014, 136, 2583. http://dx.doi.org/10.1021/jp046016j

7. Cheng, P.; Zhou, J.; Qing, Z.; Kang, W.; Liu, S.; Liu, W.; Xie, H.; Zeng, J. Bioorg. Med. Chem. Lett. 2014, 24, 2712. http://dx.doi.org/10.1016/j.bmcl.2014.04.047

8. Nagesh, H. N.; Naidu, K. M.; Rao, D. H.; Sridevi, J. P.; Sriram, D.; Yogeeswari, P.; Chandra Sekhar, K. V. Bioorg. Med. Chem. Lett. 2013, 23, 6805.

http://dx.doi.org/10.1016/j.bmcl.2013.10.016

9. Ishikawa, T. Med. Res. Rev. 2001, 21, 61. http://dx.doi.org/10.1002/(ISSN)1098-1128

10. Li, K.; Frankowski, K. J.; Frick, D. N. J. Med. Chem. 2012, 55, 3319. http://dx.doi.org/10.1021/jm300021v

11. Dubost, E.; Dumas, N.; Fossey, C.; Magnelli, R.; Butt-Gueulle, S.; Ballandonne, C.; Caignard, D. H.; Dulin, F.; de Oliveira Santos, J. S.; Millet, P.; Charnay, Y.; Rault, S.; Cailly, T.; Fabis, F. J. Med. Chem. 2012, 55, 9693. http://dx.doi.org/10.1021/jm300943r

12. Stevens, N.; O'Connor, N.; Vishwasrao, H.; Samaroo, D.; Kandel, E. R.; Akins, D. L.; Drain, C. M.; Turro, N. J. J. Am. Chem. Soc. 2008, 130, 7182. http://dx.doi.org/10.1021/ja8008924

13. Zhang, J.; Lakowicz, J. R. J. Phys. Chem. B. 2005, 109, 8701. http://dx.doi.org/10.1021/jp046016j

14. Tobisu, M.; Koh, K.; Furukawa, T.; Chatani, N. Angew. Chem., Int. Ed. 2012, 51, 11363. http://dx.doi.org/10.1002/anie.201206115 
15. Cheng, Y.; Jiang, H.; Zhang, Y.; Yu, S. Org. Lett. 2013, 15, 5520. http://dx.doi.org/10.1021/o14026827

16. Wang, Q.; Dong, X.; Xiao, T.; Zhou, L. Org. Lett. 2013, 15, 4846. http://dx.doi.org/10.1021/o14022589

17. Zhang, B.; Mück-Lichtenfeld, C.; Daniliuc, C. G.; Studer, A. Angew. Chem., Int. Ed. 2013, $52,10792$. http://dx.doi.org/10.1002/anie.201306082

18. Leifert, D.; Daniliuc, C. G.; Studer, A. Org. Lett. 2013, 15, 6286. http://dx.doi.org/10.1021/o1403147v

19. Pan., C. D.; Zhang, H. L.; Han, J.; Cheng, Y. X.; Zhu. C. J.; Chem. Commun. 2015, 51, 3786. http://dx.doi.org/10.1039/c4cc10015h

20. Sha, W. X.; Yu, J. T.; Jiang, Y.; Yang. H.; Cheng, J. Chem. Commun. 2014, 50, 9179. http://dx.doi.org/10.1039/c4cc03304c

21. Jiang, H.; Cheng, Y.; Wang, R.; Zheng, M.; Zhang, Y.; Yu, S. Angew. Chem., Int. Ed. 2013, $52,13289$.

http://dx.doi.org/10.1002/anie.201308376

22. Lenoir, I.; Smith, M. L. J. Chem. Soc. Perkin Trans. 1, 2000, 641. http://dx.doi.org/10.1039/A908630G

23. Zhang, B.; Daniliuc, C. G.; Studer, A. Org. Lett. 2014, 16, 250. http://dx.doi.org/10.1021/o1403256e

24. Gu, L. J.; Jin, C.; Liu, J. Y.; Ding, H. Y.; Fan, B. M. Chem. Commun. 2014, 50, 4643. http://dx.doi.org/10.1039/C4CC01487A

25. Liu, J.; Fan, C.; Yin, H.; Qin, C.; Zhang, G.; Zhang, X.; Yi, H.; Lei, A. Chem. Commun. 2014, 50, 2145.

http://dx.doi.org/10.1039/C3CC49026B

26. Xiao, T.; Li, L.; Lin, G.; Wang, Q.; Zhang, P.; Mao, Z.; Zhou, L. Green Chem. 2014, 16, 2418.

http://dx.doi.org/10.1039/C3GC42517G

27. Fang, H.; Zhao, J.; Qian, P.; Han, J. L.; Pan, Y. Asian J. Org. Chem. 2014, 3, 1266. http://dx.doi.org/10.1002/ajoc.201402169

28. Guo, W.; Li, S.; Tang, L.; Li, M.; Wen, L.; Chen, C. Org. Lett. 2015, 17, 1232. http://dx.doi.org/10.1021/acs.orglett.5b00197

29. Anzini, M.; Cappelli, A.; Vomero, S.; Giorgi, G.; Langer, T.; Hamon, M.; Merahi, N.; Emerit, B. M.; Cagnotto, A.; Skorupska, M.; Mennini, T.; Pinto, J. C. J. Med. Chem. 1995, $38,2692$.

http://dx.doi.org/10.1021/jm00014a021

30. Waghray, D.; Zhang, J.; Jacobs, J.; Nulens, W.; Basarić, N.; Meervelt, L. V.; Dehaen, W. J. Org. Chem. 2012, 77, 10176.

http://dx.doi.org/10.1021/jo301814m 